\title{
Essential Diagnostics - The Role of the Department of Biomedical Research of the Royal Tropical Institute in the 21 st Century
}

\author{
Paul R Klatser \\ Department of Biomedical Research, Royal Tropical Institute, Meibergdreef 39, 1105 AZ Amsterdam, \\ The Netherlands
}

In the light of emerging and overlooked infectious diseases and widespread drug resistance, diagnostics have become increasingly important in supporting surveillance, disease control and outbreak management programs.

In many low-income countries the diagnostic service has been a neglected part of health care, often lacking quantity and quality or even non-existing at all.

High-income countries have exploited few of their advanced technical abilities for the much-needed development of low-cost, rapid diagnostic tests to improve the accuracy of diagnosis and accelerate the start of appropriate treatment. As is now also recognized by World Healt Organization, investment in the development of affordable diagnostic tools is urgently needed to further our ability to control a variety of diseases that form a major threat to humanity.

The Royal Tropical Institute's Department of Biomedical Research aims to contribute to the health of people living in the tropics. To this end, its multidisciplinary group of experts focuses on the diagnosis of diseases that are major health problems in low-income countries. In partnership we develop, improve and evaluate simple and cheap diagnostic tests, and perform epidemiological studies. Moreover, we advice and support others - especially those in developing countries - in their efforts to diagnose infectious diseases.

Key words: Royal Tropical Institute - essential diagnostics

Biomedical diagnostics like laboratory tests play a crucial role in the effective diagnosis and treatment of patients in health care systems all over the world. Essential laboratory tests are complementary to the conventional physical examination by doctors and nurses to confirm the possible diagnoses for a correct and timely treatment. Diagnostic tests have recently gained impetus because of the shortcomings of the syndromic approach of diagnosis; e.g. leptospirosis is often falsely diagnosed as dengue haemoraghic fever. Also the increase in the number of infections with atypical presentations or locations, such as is the case with tuberculosis in conjunction with human immunedeficiency virus and the occurrence of asymptomatic infections, such as may be the case with sexual transmission diseases in women, make diagnostic testing vital.

To improve effective treatment, many governments of developing countries have adopted a national essential drugs policy. These national poli-

Fax: +31-20-697.1841. E-mail: p.klatser@ kit.nl Received 7 August 2000 Accepted 4 September 2000 cies are based on National Essential Drug Lists and Standard Treatment Guidelines specific for each country's health problems. However, the diagnoses, on which the recommended treatment in these Standard Treatment Guidelines is based, are often unreliable due to inadequate availability of appropriate and effective laboratory test results. These unreliable diagnoses thus seriously undermine the relevance of national essential drugs policies and their Standard Treatment Guidelines.

The reasons for the inadequate availability of relevant laboratory test results are likely to be manifold. These constraints include the lack of diagnostic assays, the selection of inappropriate laboratory diagnostics by health authorities, limited maintenance of diagnostic equipment, incorrect use of diagnostic equipment and tests by health workers, limited possibilities for quality control and assurance, patient expectations and the level of patient fees that make laboratory tests unaffordable for some segments of the population.

Considerable funds are likely to be spent on laboratory diagnostics by health authorities as well as households. If laboratory diagnostics are not appropriate and not effective they will worsen the individual patients' and the community's poverty 
by depleting scarce resources while not improving health.

Clearly, there is a need for the development of management tools to improve the effectiveness of laboratory diagnosis and at the same time there is a necessity to develop affordable, simple and robust point of care diagnostic tests which may be performed outside the laboratory. Notably the development of near-patient diagnostic assays is an unmet need. It is neither met by the private sector, nor by the public sector.

The development of simple but robust assays is expensive, existing production lines are often not equipped for their production and the marketing and distribution channels in low-income countries may be problematic. All this cumulates in low profit margins and thus the private sector is not likely to jump in because of the likelihood of no return of their investment. The public sector, such as universities and research institutes consider the development of diagnostics not as their reason of being, with few scientific incentives and limited funding possibilities. Furthermore, they are usually not familiar with quality assurance and quality control standards, neither with scaling-up.

But even if the need of development of simple diagnostics can be met, there are still other problems to overcome, relating to need assessment, patent and regulatory issues, reference materials and evaluation.

Need assessment in developing countries can be difficult because reliable data on incidences are missing. This may result in a chicken and egg situation, because being short of such information is often the consequence of a lack of diagnostics. Patents on key technologies and materials necessary to develop simple assays may be a complicating factor in the development of an assay and may determine to a large part its market price. For example, up-front payments of up to $\$ 250,000$ and royalties up to $20 \%$ of the selling price are not uncommon for a license on key patents related to immunochromatographybased assays. There are in general no legal evaluation guidelines for diagnostic test performance and primary reference materials are essentially not available. The specific problem areas encountered in attempting to apply economic evaluation of diagnostic procedures comprise the difficulty to define homogeneous patient groups for analysis and measuring the diagnostic accuracy.

Obviously, there is a need for essential diagnostics, but there are serious problems to overcome. Are we able to solve these problems? Yes, I think we can. What we would need is an essential diagnostics programme, similar to the Essential Drug Programme of World Health Organization. Such a programme could provide guidelines for priority setting in diagnostic tests, promote and facilitate the access to diagnostics (i.e. the development and marketing), assure the quality of tests and finally ensure a rational use of diagnostics with a link to standard treatment regimens. An Essential Diagnostics Programme would provide the comprehensive strategy and co-ordination necessary to forge partnerships between public and private stakeholders. Such partnerships are imperative to address some of the above-mentioned issues, notably the development of diagnostics, which determines to a large part the access. Cost-containment pressures in industrialised countries have divided the market in two sectors: automation and near-patient care. Since point-of care diagnostics for use in industrialised countries share a common base in technology with the simple tests needed in low-income countries, it is now an opportune time to benefit from this development and seek partnership with industries.

The Royal Tropical Institute's (KIT) Department of Biomedical Research (BO) aims to contribute to the health of people living in the tropics. To this end, its multidisciplinary group of experts focuses on the diagnosis of diseases that are major health problems in low-income countries. In partnership with developing countries and industry we develop, improve and evaluate simple and cheap diagnostic tests, and perform epidemiological studies. Moreover, we advice and support others - especially those in developing countries - in their efforts to diagnose and control infectious diseases. For a large part BO's work consists of basic research addressing the technical hurdles in diagnostics development process. For many years now BO has successfully collaborated with industry in the development of diagnostics. The world-wide used Lepto-Dipstick, a simple and cheap test for the diagnosis of leptospirosis and the Leish-DAT, a simple direct agglutination test for the diagnosis of visceral leishmaniasis, are illustrative for the outcome of these collaborations. In this respect, it should be stressed that the KIT is a non-profit organisation that works on a cost-recovery basis and does not make profits.

Apart from the more basic research, KIT-BO is involved in comparative trials and cost-effectiveness, utility, and impact studies related to diagnostics.

In the 21 st century KIT-BO will continue its efforts in biomedical research aimed at the development of essential diagnostics, thus trying to contribute to the health of people living in low-income countries. It is hoped that KIT-BO can perform its task as part of a concerted action of all stakeholders. Essential diagnostics are not a luxury, but a need. 